\title{
COURANT MORPHISMS AND MOMENT MAPS
}

\author{
Henrique Bursztyn, David Iglesias Ponte, and Pavol Ševera
}

\begin{abstract}
We study Hamiltonian spaces associated with pairs $(E, A)$, where $E$ is a Courant algebroid and $A \subset E$ is a Dirac structure. These spaces are defined in terms of morphisms of Courant algebroids with suitable compatibility conditions. Several of their properties are discussed, including a reduction procedure. This set-up encompasses familiar moment map theories, such as group-valued moment maps, and it provides an intrinsic approach from which different geometrical descriptions of moment maps can be naturally derived. As an application, we discuss the relationship between quasi-Poisson and presymplectic groupoids.
\end{abstract}

\section{Introduction}

In this paper, we study Hamiltonian spaces naturally associated with Manin pairs, i.e. pairs $(E, A)$, where $E$ is a Courant algebroid, and $A \subset E$ is a Dirac structure. As we will see, these objects retain many of the features of ordinary Hamiltonian spaces, including a reduction procedure, and several moment map theories can be expressed in these terms.

Our main motivation to consider this general set-up comes from the theory of Hamiltonian spaces with group-valued moment maps (as in [17] and $[3,4]$ ) or, more generally, of moment maps with values in homogeneous spaces $D / G$. There are two geometrical formulations of $D / G$-valued moment maps: The original one of Alekseev and Kosmann-Schwarzbach [2] is given in terms of quasi-Poisson actions, whereas [8] presents an alternative approach based on morphisms of Dirac manifolds. (In the special case of $G$-valued moment maps, these two distinct viewpoints can be found in [3] and [4], respectively.) Both approaches originate from a common starting point, namely a Manin pair, but each one requires an additional, noncanonical piece of information; depending on this extra choice, Hamiltonian spaces are either described in terms of quasi-Poisson or twisted Dirac structures. As proven in [8, Sec. 6], these two viewpoints, despite resorting to different geometrical structures, produce isomorphic categories of Hamiltonian spaces, regardless of any of the extra data used. This raises the question of whether there is an intrinsic notion of Hamiltonian space associated with a Manin pair, requiring no extra data at all, that would naturally recover the formulations in [2] and [8] once suitable additional choices are made. The main goal of this paper is to present such intrinsic notion and to study its properties, showing that it offers a clear conceptual explanation for the equivalence between the quasi-Poisson and Dirac geometric approaches to moment maps.

We organize the paper as follows. We review the basics of Courant algebroids and Dirac structures [16] in Section 2, where we also introduce the notion of morphism of Manin pairs, which is central in this paper. In Section 3, we define Hamiltonian

Received by the editors January 13, 2008. 
spaces associated with Manin pairs $(E, A)$ as special examples of morphisms of Manin pairs. We focus on two possible scenarios: when $E=A \oplus A^{*}$ is the double of a Lie quasi-bialgebroid [21], then the Hamiltonian spaces for $(E, A)$ can be naturally identified with Hamiltonian quasi-Poisson spaces (in the sense of [14], see also [15]); on the other hand, when $E=T S \oplus T^{*} S$ is a Courant algebroid defined by a closed 3 -form on a manifold $S$ [26], then $A \subset T S \oplus T^{*} S$ is a Dirac structure on $S$, and the Hamiltonian spaces for $(E, A)$ are identified with Hamiltonian spaces defined by strong Dirac maps into $S$ (as considered in $[1,7,8]$ ). Combining these two possible "incarnations" of the intrinsic Hamiltonian spaces for $(E, A)$, we arrive at a functorial correspondence between moment maps in quasi-Poisson and Dirac geometries, which gives a new, more general viewpoint to the results in [8, Sec. 6.2] (as well as [1, Sec. 5.4] and [7, Sec. 3.5]). We also discuss Poisson spaces obtained via reduction of Hamiltonian spaces for $(E, A)$, showing that they agree with quasi-Poisson and Dirac reductions in specific situations. Finally, in Section 4, we apply the correspondence between Hamiltonian spaces in quasi-Poisson and Dirac geometries to find an explicit construction relating presymplectic groupoids [10] and quasi-Poisson groupoids [14].

\section{Preliminaries}

2.1. Courant algebroids and Manin pairs. A Courant algebroid [16] over a manifold $S$ is a vector bundle $E \rightarrow S$ equipped with a nondegenerate symmetric bilinear form $\langle\cdot, \cdot\rangle$ on the bundle, a bundle map $\rho: E \rightarrow T S$ and a bilinear bracket $\llbracket \cdot, \cdot \rrbracket$ on $\Gamma(E)$ such that $\forall e, e_{1}, e_{2}, e_{3} \in \Gamma(E), f \in C^{\infty}(S)$, the following holds:

c1) $\llbracket e_{1}, \llbracket e_{2}, e_{3} \rrbracket \rrbracket=\llbracket \llbracket e_{1}, e_{2} \rrbracket, e_{3} \rrbracket+\llbracket e_{2}, \llbracket e_{1}, e_{3} \rrbracket \rrbracket ;$

c2) $\llbracket e, e \rrbracket=\rho^{*} d\langle e, e\rangle$, where we use $\langle\cdot, \cdot\rangle$ to identify $E \cong E^{*}$;

c3) $\mathcal{L}_{\rho(e)}\left\langle e_{1}, e_{2}\right\rangle=\left\langle\llbracket e, e_{1} \rrbracket, e_{2}\right\rangle+\left\langle e_{1}, \llbracket e, e_{2} \rrbracket\right\rangle$

c4) $\rho\left(\llbracket e_{1}, e_{2} \rrbracket\right)=\left[\rho\left(e_{1}\right), \rho\left(e_{2}\right)\right]$;

c5) $\llbracket e_{1}, f e_{2} \rrbracket=f \llbracket e_{1}, e_{2} \rrbracket+\left(\mathcal{L}_{\rho\left(e_{1}\right)} f\right) e_{2}$.

A Courant algebroid is denoted by the quadruple $(E,\langle\cdot, \cdot\rangle, \llbracket \cdot, \cdot \rrbracket, \rho)$, or simply by $E$ if there is no risk of confusion. We use the notation $\bar{E}$ for the Courant algebroid $(E,-\langle\cdot, \cdot\rangle, \llbracket \cdot, \cdot \rrbracket, \rho)$.

We recall some properties of Courant algebroids for later use. First, $c 2)$ and $c 4$ ) imply that

$$
\rho \circ \rho^{*}=0
$$

where we identify $E \cong E^{*}$ via $\langle\cdot, \cdot\rangle$. On the other hand, given 1-forms $\beta, \beta^{\prime} \in \Omega^{1}(S)$, we have

$$
\llbracket \rho^{*} \beta, \rho^{*} \beta^{\prime} \rrbracket=0 .
$$

To verify (2), it suffices to show that $\left\langle\llbracket \rho^{*} \beta, \rho^{*} \beta^{\prime} \rrbracket, e\right\rangle=0, \forall e \in \Gamma(E)$, and this follows from $c 3$ ) combined with $c 4$ ) and (1).

A subbundle $A \subset E$ is called an almost Dirac structure if it is Lagrangian with respect to $\langle\cdot, \cdot\rangle$ (i.e. both isotropic and coisotropic), and it is a Dirac structure if, in addition, it is integrable, that is, $\Gamma(A)$ is closed under $\llbracket \cdot, \cdot \rrbracket$. The restrictions of the anchor and Courant bracket to any Dirac structure $A$ make it into a Lie algebroid. We denote the restricted bracket by $[\cdot, \cdot]_{A}:=\llbracket \cdot,\left.\cdot \rrbracket\right|_{\Gamma(A)}$. Pairs $(E, A)$, where $E$ is a 
Courant algebroid over $S$ and $A \subset E$ is a Dirac structure, are central objects in this paper. We refer to them as Manin pairs over $S .^{1}$

Example 2.1. A Manin pair over a point is a pair $(\mathfrak{d}, \mathfrak{g})[2]$, where $\mathfrak{d}$ is a $2 n$ dimensional Lie algebra equipped with an Ad-invariant inner product, and $\mathfrak{g} \subset \mathfrak{d}$ is a Lagrangian Lie subalgebra.

Example 2.2. Let $(\mathfrak{d}, \mathfrak{g})$ be a Manin pair (over a point) as in Example 2.1. Following [2], let $D$ and $G$ be connected Lie groups integrating $\mathfrak{d}$ and $\mathfrak{g}$, and assume that $G$ is a closed subgroup of $D$. Let $S:=D / G$ be the quotient with respect to right multiplication. Then the action of $D$ on itself by left multiplication induces a $D$-action on $S$, called the dressing action. The pairing and bracket on $\mathfrak{d}$ give rise to a natural Courant algebroid structure on the trivial bundle $\mathfrak{d}_{S}:=\mathfrak{d} \times S$ over $S[5,23]$ (c.f. [8, Sec. 3]) for which the anchor is the infinitesimal dressing action $\mathfrak{d}_{S} \rightarrow T S$. The subbundle $\mathfrak{g}_{S}=\mathfrak{g} \times S$ defines a Dirac structure, i.e. $\left(\mathfrak{d}_{S}, \mathfrak{g}_{S}\right)$ is a Manin pair over $S$.

Example 2.3. Let $\mathbb{T} S:=T S \oplus T^{*} S$, equipped with pairing $\left\langle(v, \alpha),\left(v^{\prime}, \alpha^{\prime}\right)\right\rangle=\alpha^{\prime}(v)+$ $\alpha\left(v^{\prime}\right)$. A closed 3-form $\phi_{S} \in \Omega^{3}(S)$ defines the Courant bracket [26]

$$
\llbracket(v, \alpha),\left(v^{\prime}, \alpha^{\prime}\right) \rrbracket:=\left(\left[v, v^{\prime}\right], \mathcal{L}_{v} \alpha^{\prime}-i_{v^{\prime}} d \alpha+i_{v^{\prime}} i_{v} \phi_{S}\right),
$$

making $\mathbb{T} S$ a Courant algebroid with anchor given by the natural projection $\mathbb{T} S \rightarrow T S$. When $\phi_{S}=0$, we refer to this Courant structure on $\mathbb{T} S$ as standard.

Let $(E, A)$ be a Manin pair over $S$. Consider the exact sequence $0 \rightarrow A \rightarrow E \rightarrow$ $E / A \cong A^{*} \rightarrow 0$. We can always fix an isotropic splitting of this sequence, i.e., a splitting

$$
j: A^{*} \rightarrow E
$$

whose image is isotropic (see e.g. [8, App. 2]). This defines a cobracket $F_{j}: \Gamma(A) \rightarrow$ $\wedge^{2} \Gamma(A)$, a bundle map $\rho_{A^{*}}^{j}: A^{*} \rightarrow T M$ and a 3-tensor $\chi_{j} \in \Gamma\left(\wedge^{3} A\right)$ by

$$
F_{j}(a)\left(\xi_{1}, \xi_{2}\right)=\left\langle\llbracket j\left(\xi_{1}\right), j\left(\xi_{2}\right) \rrbracket, a\right\rangle, \quad \rho_{A^{*}}^{j}=\rho \circ j, \quad \chi_{j}\left(\xi_{1}, \xi_{2}, \xi_{3}\right)=\left\langle\llbracket j\left(\xi_{1}\right), j\left(\xi_{2}\right) \rrbracket, j\left(\xi_{3}\right)\right\rangle,
$$

where $a \in \Gamma(A), \xi_{1}, \xi_{2}, \xi_{3} \in \Gamma\left(A^{*}\right)$, making $A$ into a Lie quasi-bialgebroid [14, 21] in such a way that $E$ is naturally identified with the "double" Courant algebroid $A \oplus A^{*}[21]$. Equivalently, $F_{j}$ and $\rho_{A^{*}}^{j}$ can be combined into a degree 1 derivation $d_{A^{*}}: \Gamma\left(\wedge^{\bullet} A\right) \rightarrow \Gamma\left(\wedge^{\bullet+1} A\right)$ such that $d_{A^{*}}^{2}=\left[\chi_{j}, \cdot\right]_{A}$ and $d_{A^{*}} \chi_{j}=0$.

\subsection{Dirac structures with support.}

Definition 2.4. Given a Courant algebroid $(E,\langle\cdot, \cdot\rangle, \llbracket \cdot, \cdot \rrbracket, \rho)$ over a manifold $M$ and a submanifold $\iota: Q \hookrightarrow M$, a Dirac structure supported on $Q$ (see $[5,25])$ is a subbundle $K \subset \iota^{*} E=\left.E\right|_{Q}$ such that $K_{x} \subset E_{x}$ is Lagrangian for all $x \in Q$ and the following two conditions hold:

d1) $K$ is compatible with the anchor, that is, $\rho(K) \subset T Q$;

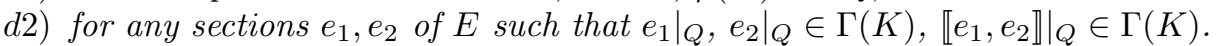
If only $d 1)$ is satisfied, we refer to an almost Dirac structure supported on $Q$.

\footnotetext{
${ }^{1}$ Lagrangian subbundles $A \subset E$ exist if and only if the pairing $\langle\cdot, \cdot\rangle$ has split signature $(n, n)$. The results in this paper remain valid when $A \subset E$ is only required to be maximal isotropic, in which case there is no signature requirement on $\langle\cdot, \cdot\rangle$.
} 
Remark 2.5. The Leibniz rule for Courant algebroids (condition c5)) shows that if $e_{1}$ and $e_{2}$ satisfy $\left.e_{1}\right|_{Q}=\left.e_{2}\right|_{Q}$, then $\llbracket e,\left.e_{1} \rrbracket\right|_{Q}=\llbracket e,\left.e_{2} \rrbracket\right|_{Q}$ for all $e \in \Gamma(E)$ with $\left.e\right|_{Q}$ in $K$. Hence it suffices to check $d 2)$ for a set of sections of $E$ whose restrictions to $Q$ locally generate $K$.

Example 2.6. Let $Q$ be a submanifold of $M$, and denote by $N Q$ its normal bundle. We consider $\mathbb{T} M$ equipped with its standard Courant algebroid structure. Then the subbundle $\left.T Q \oplus N Q \subset \mathbb{T} M\right|_{Q}$ is a Dirac structure supported on $Q$.

Example 2.7. Let $X$ and $X^{\prime}$ be smooth manifolds, and let $f: X \rightarrow X^{\prime}$ be a smooth map. We consider the standard Courant algebroids $\mathbb{T} X^{\prime}$ and $\overline{\mathbb{T} X}$, and the product Courant algebroid $E=\mathbb{T} X^{\prime} \times \overline{\mathbb{T} X}$ over $M=X^{\prime} \times X$. Then

$$
\Gamma_{f}:=\left\{\left((d f(v), \alpha),\left(v, f^{*} \alpha\right)\right) \mid v \in T_{x} X \text { and } \alpha \in T_{f(x)}^{*} X^{\prime}, x \in X\right\}
$$

is a Dirac structure in $E$ supported on $Q=\operatorname{graph}(f) \subset M$.

If $E, E^{\prime}$ are Courant algebroids over $X, X^{\prime}$, then a Courant algebroid morphism between $E$ and $E^{\prime}$ is a Dirac structure in $E \times \bar{E}^{\prime}$ supported on $\operatorname{graph}(f)$, where $f: X \rightarrow X^{\prime}$ is a smooth map (see e.g. [5] and Remark 2.12).

We have the following natural correspondence.

Proposition 2.8. Let $f: Q \hookrightarrow M$ be an embedding and $\phi \in \Omega^{3}(M)$ be a closed 3-form. Let $\operatorname{Dir}(Q)$ denote the set of Dirac structures in $\mathbb{T} Q$, integrable relative to $f^{*} \phi$, and $\operatorname{Dir}(M)_{f(Q)}$ denote the set of Dirac structures in $\mathbb{T} M$ supported on $f(Q)$, integrable relative to $\phi$. Then these sets are in bijection via the maps

$$
\begin{aligned}
& \mathfrak{F}_{f}: \operatorname{Dir}(Q) \rightarrow \operatorname{Dir}(M)_{f(Q)}, \mathfrak{F}_{f}(L)_{f(x)}:=\left\{(d f(u), \beta) \mid\left(u, f^{*} \beta\right) \in L_{x}\right\}, \\
& \mathfrak{B}_{f}: \operatorname{Dir}(M)_{f(Q)} \rightarrow \operatorname{Dir}(Q), \mathfrak{B}_{f}(L)_{x}:=\left\{\left(u, f^{*} \beta\right) \mid(d f(u), \beta) \in L_{f(x)}\right\},
\end{aligned}
$$

which are inverses of one another.

Proof. Consider the injective bundle map $\psi: \mathbb{T} Q \rightarrow f^{*} T M \oplus T^{*} Q, \psi(u, \alpha)=$ $(d f(u), \alpha)$, and the surjective bundle map $p: f^{*} \mathbb{T} M \rightarrow f^{*} T M \oplus T^{*} Q, p(v, \beta)=$ $\left(v, f^{*} \beta\right)$. Then $\mathfrak{F}_{f}(L)=p^{-1}(\psi(L))$ is a smooth vector bundle over $f(Q)$, which one can directly check to be maximal isotropic. To verify integrability, consider sections $(v, \beta),\left(v^{\prime}, \beta^{\prime}\right)$ of $\mathbb{T} M$ whose restrictions to $f(Q)$ lie in $\mathfrak{F}_{f}(L)$. Let $u, u^{\prime} \in$ $\Gamma(T Q)$ be such that $\left.v\right|_{f(Q)}=d f(u),\left.v^{\prime}\right|_{f(Q)}=d f\left(u^{\prime}\right)$, and consider $\llbracket(v, \beta),\left(v^{\prime}, \beta^{\prime}\right) \rrbracket=$ $\left(\left[v, v^{\prime}\right], \mathcal{L}_{v} \beta^{\prime}-i_{v^{\prime}} d \beta+i_{v \wedge v^{\prime}} \phi\right)$. Then $\left.\left[v, v^{\prime}\right]\right|_{f(Q)}=d f\left(\left[u, u^{\prime}\right]\right) \in T f(Q)$ and, similarly, $f^{*} \mathcal{L}_{v} \beta^{\prime}=\mathcal{L}_{u} f^{*} \beta^{\prime}, f^{*} i_{v^{\prime}} d \beta=i_{u^{\prime}} d f^{*} \beta$, and $f^{*}\left(i_{v \wedge v^{\prime}} \phi\right)=i_{u \wedge u^{\prime}} f^{*} \phi$. Using these relations and the integrability of $L$, it follows that $\llbracket(v, \beta),\left.\left(v^{\prime}, \beta^{\prime}\right) \rrbracket\right|_{f(Q)} \in \mathfrak{F}_{f}(L)$. The map $\mathfrak{B}_{f}$ can be treated analogously.

Finally, for $L \in \operatorname{Dir}(M)_{f(Q)}$, one can check that $\mathfrak{F}_{f} \circ \mathfrak{B}_{f}(L)=L$ if and only if $\operatorname{pr}_{T M}(L) \subseteq T f(Q)$, which holds since $L$ is supported on $f(Q)$. Similarly, for $L \in \operatorname{Dir}(Q), \mathfrak{B}_{f} \circ \mathfrak{F}_{f}(L)=L$ if and only if $\operatorname{ker}(d f) \subseteq T Q \cap L$, which holds since $f$ is an embedding.

2.3. Morphisms of Manin pairs. Let $\left(E_{i}, A_{i}\right)$ be a Manin pair over $S_{i}, i=1,2$. We use the pairing in $E_{i}$ to identify $E_{i} / A_{i} \cong A_{i}^{*}$, and denote by $p_{i}: E_{i} \rightarrow A_{i}^{*}$ the natural projection. 
Definition 2.9. A morphism of Manin pairs $\left(E_{1}, A_{1}\right) \rightarrow\left(E_{2}, A_{2}\right)$ is a Dirac structure $K$ in $E_{1} \times \bar{E}_{2}$ with support on the graph of a smooth map $J: S_{1} \rightarrow S_{2}$ (i.e., a Courant algebroid morphism $E_{1} \rightarrow E_{2}$ ), such that the image of $K$ under the projection $\left(p_{1}, p_{2}\right): E_{1} \times E_{2} \rightarrow A_{1}^{*} \times A_{2}^{*}$ is the graph of a bundle map $A_{1}^{*} \rightarrow J^{*} A_{2}^{*}$.

Composition of these morphisms is by definition composition of relations (see also Rem. 2.12, where the morphisms are interpreted as maps, and the composition becomes just composition of maps).

The next proposition gives a more explicit characterization of morphisms of Manin pairs.

Proposition 2.10. Let $\left(E_{i}, A_{i}\right)$ be a Manin pair over $S_{i}, i=1,2$, and let $J: S_{1} \rightarrow S_{2}$ be a smooth map. A Dirac structure $K$ in $E_{1} \times \bar{E}_{2}$ with support on $\operatorname{graph}(J)$ is a morphism of Manin pairs $\left(E_{1}, A_{1}\right) \rightarrow\left(E_{2}, A_{2}\right)$ if and only if

i) $K \cap A_{1}=\{0\}$,

ii) $K \cap\left(A_{1} \oplus J^{*} E_{2}\right)$ projects onto $J^{*} A_{2}$ under the natural projection $E_{1} \oplus J^{*} E_{2} \rightarrow$ $J^{*} E_{2}$.

In other words, $i)$ and $i i)$ say that the projection $E_{1} \oplus J^{*} E_{2} \rightarrow J^{*} E_{2}$ restricted to $K \cap\left(A_{1} \oplus J^{*} E_{2}\right)$ is an isomorphism onto $J^{*} A_{2}$.

Proof. Let us consider the projection $p=\left(p_{1}, p_{2}\right): E_{1} \times E_{2} \rightarrow A_{1}^{*} \times A_{2}^{*}$, and let $R=p(K) \subset A_{1}^{*} \oplus J^{*} A_{2}^{*}$. We must show that conditions $i$ ) and $i i$ ) are equivalent to $R$ being the graph of a bundle map $A_{1}^{*} \rightarrow J^{*} A_{2}^{*}$, or, equivalently, that $R$ projects isomorphically onto $A_{1}^{*}$.

The projection of an element $r=p(k) \in R$ on $A_{1}^{*}$ is zero if and only if $k \in$ $K \cap\left(A_{1} \times E_{2}\right)$. In this case, $r=0$ if and only if its projection on $A_{2}^{*}$ is zero, which is equivalent to the projection of $k$ on $E_{2}$ lying in $A_{2}$. Hence $R$ projects injectively on $A_{1}^{*}$ if and only if $\left.i i\right)$ holds. If the projection of $R$ on $A_{1}^{*}$ is also onto, then $i$ ) must hold: given $a \in K \cap A_{1}$ and any $\xi \in A_{1}^{*}$, there is a $k \in K$ with $p_{1}(k)=\xi$, and since $K$ is isotropic, one has $\langle k, a\rangle=\xi(a)=0$. This implies that $a=0$. Conversely, a dimension count shows that the projection of $R$ on $A_{1}^{*}$ is onto if and only if $\operatorname{rank}\left(K \cap\left(A_{1} \times E_{2}\right)\right)=\operatorname{rank}\left(A_{2}\right)$, which follows from $\left.i\right)$ and $\left.i i\right)$.

Example 2.11. Let $S_{1}, S_{2}$ be manifolds and $f: S_{1} \rightarrow S_{2}$ a smooth map. Fix a closed 3-form $\phi \in \Omega^{3}\left(S_{2}\right)$, and consider the Courant algebroids $\mathbb{T} S_{1}, \mathbb{T} S_{2}$, with brackets defined by the 3-forms $f^{*} \phi, \phi$, respectively (see Example 2.3). There is a natural Dirac structure $K$ on $\mathbb{T} S_{1} \times \overline{\mathbb{T}}_{2}$ supported on $\operatorname{graph}(f)$ (c.f. (5)),

$$
K_{(x, f(x))}=\left\{\left(\left(X, f^{*} \beta\right),(d f(X), \beta)\right) \mid X \in T_{x} S_{1}, \beta \in T_{f(x)}^{*} S_{2}\right\} .
$$

Let $L_{i} \subset \mathbb{T} S_{i}$ be Dirac structures, so that $\left(\mathbb{T} S_{i}, L_{i}\right)$ is a Manin pair, $i=1,2$. Then property i) in Prop. 2.10 amounts to the condition $\operatorname{ker}(d f) \cap\left(T S_{1} \cap L_{1}\right)=\{0\}$, whereas ii) says that $f$ is a forward Dirac map [11]. Hence $K$ defines a morphism of Manin pairs $\left(\mathbb{T} S_{1}, L_{1}\right) \rightarrow\left(\mathbb{T} S_{2}, L_{2}\right)$ if and only if $f$ is a strong Dirac map as in $[1,7,8]$ (see also Section 3.2). More generally, one can fix a 2-form $\omega \in \Omega^{2}\left(S_{1}\right)$, define the Courant bracket on $\mathbb{T} S_{1}$ using $f^{*} \phi+d \omega$, and check that $K_{(x, f(x))}=\left\{\left(\left(X, f^{*} \beta-\right.\right.\right.$ $\left.\left.\left.i_{X} \omega\right),(d f(X), \beta)\right) \mid X \in T_{x} S_{1}, \beta \in T_{f(x)}^{*} S_{2}\right\}$ is still a Dirac structure. Then $K$ defines a morphism of Manin pairs if and only if $(f, \omega)$ is a strong Dirac morphism as in $[1$, Sec. 2.2]. 
Remark 2.12 (Super-geometric viewpoint). The notion of morphism of Manin pairs in Def. 2.9 has a natural interpretation in the framework of Gerstenhaber algebras, or equivalently in terms of odd Poisson structures, as we now briefly outline.

As observed in [24], Manin pairs (E,A) are in one-to-one correspondence with principal $\mathbb{R}[2]$-bundles $P \rightarrow A^{*}[1]$ (in the category of graded manifolds) equipped with a $\mathbb{R}[2]$-invariant Poisson structure on $P$ of degree -1 . A morphism between $P_{1} \rightarrow A_{1}^{*}[1]$ and $P_{2} \rightarrow A_{2}^{*}[1]$ is clear in this context: it is an $\mathbb{R}[2]$-equivariant Poisson map. When such morphisms are expressed in terms of the associated Manin pairs, one recovers Definition 2.9.

To see how this correspondence goes, given $P \rightarrow A^{*}[1]$, choose a trivialization of $P$ to $A^{*}[1] \times \mathbb{R}[2]$, so that the algebra of functions on $P$ becomes $\Gamma(\wedge A)[t]$, where $t$ (the coordinate on $\mathbb{R}[2])$ has degree 2. The Poisson structure on $P$ amounts to a Gerstenhaber bracket on $\Gamma(\wedge A)[t]$. By invariance, it descends to a Gerstenhaber bracket on $\Gamma(\wedge A)$, which defines a Lie algebroid structure on A. Fixing this Lie algebroid structure, the Gerstenhaber bracket on $\Gamma(\wedge A)[t]$ is determined by $[t, t] \in$ $\Gamma\left(\wedge^{3} A\right)$ and $[t, a] \in \Gamma(\wedge A)$, for all $a \in \wedge A$. By setting $\chi:=[t, t]$ and $d_{A^{*}}:=[t, \cdot]:$ $\Gamma\left(\wedge^{\bullet} A\right) \rightarrow \Gamma\left(\wedge^{\bullet-1} A\right)$, we define a Lie quasi-bialgebroid. We hence set $E=A \oplus$ $A^{*}$ to be the double Courant algebroid, so that $(E, A)$ is a Manin pair. Different trivializations of $P$ amount to different choices of isotropic complements of $A$ in $E$, so this procedure establishes the desired correspondence.

This correspondence can also be viewed more instrinsically, without the choice of splittings or trivializations.

First recall from [23] (concluding work of Vaintrob, Roytenberg [20] and Weinstein) that Courant algebroids $E$ are equivalently described as degree 2 symplectic non-negatively graded manifolds $\mathcal{M}$ equipped with a function $\Theta$ of degree 3 satisfying $\{\Theta, \Theta\}=0$ (c.f. [22]). Dirac structures in $E$ with support on a submanifold correspond to the Lagrangian submanifolds of $\mathcal{M}$ on which the degree 3 function $\Theta$ vanishes. This motivates the definition of morphism of Courant algebroids in Section 2.2.

The Manin pair associated with $P \rightarrow A^{*}[1]$ is obtained as follows. The Poisson structure on $P$ is a function $H$ on $T^{*}[2] P$, quadratic on the fibres, of total degree 3 and such that $\{H, H\}=0$. This function descends to the symplectic reduction $Y=T^{*}[2] P / / 1 \mathbb{R}[2]$ at moment value 1 , hence $Y$ determines a Courant algebroid $E$ (see [6] for more on the reduction of Courant algebroids from this perspective). The map $Y \rightarrow A^{*}[1]$ (which is a Lagrangian fibration) gives rise to a map $E \rightarrow A^{*}$, whose kernel determines $A \subset E$, defining a Manin pair $(E, A)$. Given $P_{1} \rightarrow A_{1}^{*}[1]$ and $P_{2} \rightarrow A_{2}^{*}[1]$ and a morphism $\psi: P_{1} \rightarrow P_{2}$, since $\psi$ is a Poisson map, its graph $\Gamma_{\psi}$ is coisotropic in $P_{1} \times \bar{P}_{2}$ (where $\bar{P}_{2}$ is $P_{2}$ with the opposite Poisson structure). Then the conormal bundle $N^{*}[2] \Gamma_{\psi}$ is Lagrangian in $T^{*}[2]\left(P_{1} \times P_{2}\right)$, and the function $H$ coming from the Poisson structure on $P_{1} \times \bar{P}_{2}$ vanishes on it. The reduction of $N^{*}[2] \Gamma_{\psi}$ to the symplectic quotient $Y_{1} \times \bar{Y}_{2}$ defines a Lagrangian submanifold, which corresponds to the Dirac structure $K$ of Definition 2.9.

\section{Morphisms of Manin pairs and Hamiltonian spaces}

3.1. Hamiltonian spaces. Let us consider a Manin pair $(E, A)$ over a manifold $S$. The following is the main definition of this paper (c.f. [15]). 
Definition 3.1. A Hamiltonian space for $(E, A)$ is a manifold $X$ together with a morphism of Manin pairs $(\mathbb{T} X, T X) \rightarrow(E, A)$, where $\mathbb{T} X$ is the standard Courant algebroid of $X$.

Hamiltonian spaces are denoted by triples $(X, J, K)$ (where the map $J: X \rightarrow S$ and the Dirac structure $K$ are as in Definition 2.9), and $J$ is the moment map.

A morphism between two Hamiltonian spaces $(X, J, K)$ and $\left(X^{\prime}, J^{\prime}, K^{\prime}\right)$ is a smooth map $f: X \rightarrow X^{\prime}$ such that $J(x)=J^{\prime}(f(x)), \forall x \in X$, and $\Gamma_{f} \circ K=K^{\prime}$, where $\Gamma_{f}$ is defined in (5), Example 2.7, and o denotes composition of relations, i.e.,

$$
\begin{aligned}
\left(K^{\prime}\right)_{(f(x), J(x))=} & \left\{\left(\left(u^{\prime}, \alpha^{\prime}\right), e\right) \mid\left(u^{\prime}, \alpha^{\prime}\right) \in\left(\mathbb{T} X^{\prime}\right)_{f(x)}, e \in E_{J(x)},\right. \\
& \text { and } \left.\exists u \in(T X)_{x} \text { s.t. } u^{\prime}=d f(u),\left(\left(u, f^{*}\left(\alpha^{\prime}\right)\right), e\right) \in(K)_{(x, J(x))}\right\},
\end{aligned}
$$

$\forall x \in X$, see e.g. [11, 15]. (Note that $(f(x), J(x)) \in \operatorname{graph}\left(J^{\prime}\right)$.) The category of Hamiltonian spaces associated with the Manin pair $(E, A)$ is denoted by $\overline{\mathcal{M}}(E, A)$.

Notice that the projection $\mathbb{T} X \times E \rightarrow E$ restricts to an isomorphism

$$
K_{(x, J(x))} \cap\left((T X \oplus\{0\})_{x} \times E_{J(x)}\right) \stackrel{\sim}{\longrightarrow} A_{J(x)}, \quad \forall x \in X .
$$

Example 3.2. We present two examples of Hamiltonian spaces canonically associated with any pair $(E, A)$ over $S$. Let $\Delta \subset S \times S$ be the diagonal, and consider the subbundle $\left.K \subset(\mathbb{T} S \times E)\right|_{\Delta}$ given by

$$
K_{(x, x)}:=\left\{\left((\rho(a),-\beta), a+\rho^{*} \beta\right) \mid a \in A_{x}, \beta \in\left(T^{*} S\right)_{x}\right\} \subset(\mathbb{T} S)_{x} \times E_{x}, x \in S .
$$

This is a Dirac structure in $\mathbb{T} S \times E$ supported on $\Delta$, and it defines a Hamiltonian space $(X, J, K)$ for $(E, A)$, where $X=S$ and $J=\mathrm{Id}$. Indeed, a direct check using (1) shows that $K$ is isotropic, and a dimension count gives that it has maximal rank. Condition d1) in Def. 2.4 is a consequence of (1). To verify the integrability condition $d 2)$, it suffices to consider sections of $\mathbb{T} S \times E$ of the form $\widetilde{a}=((\rho(a), 0), a), a \in \Gamma(A)$, and $\widetilde{\beta}=\left((0,-\beta), \rho^{*} \beta\right), \beta \in \Omega^{1}(S)$, and check that

$$
\llbracket \widetilde{a},\left.\widetilde{a}^{\prime} \rrbracket\right|_{\Delta}, \llbracket \widetilde{\beta},\left.\widetilde{\beta}^{\prime} \rrbracket\right|_{\Delta}, \llbracket \widetilde{a},\left.\widetilde{\beta} \rrbracket\right|_{\Delta} \in \Gamma(K),
$$

see Remark 2.5. The first case follows directly from $c 4)$. For the second case, (2) gives $\llbracket \widetilde{\beta}, \widetilde{\beta^{\prime}} \rrbracket=0$. For the last case, note that $\llbracket \widetilde{a}, \widetilde{\beta} \rrbracket=\left(\left(0,-\mathcal{L}_{\rho(a)} \beta\right), \llbracket a, \rho^{*} \beta \rrbracket\right)$. It is immediate to check (using c4) and (1)) that $\left\langle\llbracket \widetilde{a}, \widetilde{\beta} \rrbracket, \widetilde{\beta}^{\prime}\right\rangle=0, \forall \beta^{\prime}$. On the other hand, using $(3)$, for all $\widetilde{a}^{\prime}$ we have

$$
\begin{aligned}
\left\langle\llbracket \widetilde{a}, \widetilde{\beta} \rrbracket, \widetilde{a}^{\prime}\right\rangle & =-i_{\rho\left(a^{\prime}\right)} \mathcal{L}_{\rho(a)} \beta+\left\langle a^{\prime}, \llbracket a, \rho^{*} \beta \rrbracket\right\rangle \\
& =-i_{\rho\left(a^{\prime}\right)} \mathcal{L}_{\rho(a)} \beta-\left\langle\llbracket a, a^{\prime} \rrbracket, \rho^{*} \beta\right\rangle+\mathcal{L}_{\rho(a)} i_{\rho\left(a^{\prime}\right)} \beta=0,
\end{aligned}
$$

since $\left\langle\llbracket a, a^{\prime} \rrbracket, \rho^{*} \beta\right\rangle=i_{\left[\rho(a), \rho\left(a^{\prime}\right)\right]} \beta=\mathcal{L}_{\rho(a)} i_{\rho\left(a^{\prime}\right)} \beta-i_{\rho\left(a^{\prime}\right)} \mathcal{L}_{\rho(a)} \beta$. Hence, $\left\langle\llbracket \widetilde{a},\left.\widetilde{\beta} \rrbracket\right|_{\Delta}, \Gamma(K)\right\rangle$ $=0$, and since $K$ is maximal isotropic, $\llbracket \widetilde{a},\left.\widetilde{\beta} \rrbracket\right|_{\Delta} \in \Gamma(K)$.

Similarly, let $\iota: \mathcal{O} \hookrightarrow S$ be an orbit of $A$ (i.e., an integral leaf of $\rho(A) \subseteq T S$ ), and $\left.K \subset(\mathbb{T} \mathcal{O} \times E)\right|_{\operatorname{graph}(\iota)}$ be given by $K_{(x, \iota(x))}=\left\{\left(\left(\rho(a),-\iota^{*} \beta\right), a+\rho^{*} \beta\right) \mid a \in A_{x}, \beta \in\right.$ $\left.\left(T^{*} S\right)_{x}\right\}$. Then $(X, J, K)$, where $X=\mathcal{O}$ and $J=\iota$, is a Hamiltonian space for $(E, A)$.

The definition of Hamiltonian space implies the following properties:

Proposition 3.3. Let $(X, J, K)$ be a Hamiltonian space for $(E, A)$. Then: 
i) For any $a \in A_{J(x)}$, there exists a unique $u \in T_{x} X$ such that $((u, 0), a) \in$ $K_{(x, J(x))}$. Moreover, this induces a Lie algebroid action $\rho_{X}: J^{*} A \rightarrow T X$ of $A$ on $J: X \rightarrow S$ (i.e., $\rho_{X}$ is a smooth bundle map so that $d J\left(\rho_{X}(a)_{x}\right)=\rho\left(a_{J(x)}\right)$ and the induced map $\rho_{X}: \Gamma(A) \rightarrow \mathfrak{X}(X)$ preserves Lie brackets.)

ii) For any $\alpha \in T_{x}^{*} X$, there exists $e \in E_{J(x)}$ and $u \in T_{x} X$ such that $((u, \alpha), e) \in$ $K_{(x, J(x))}$. In addition, $\rho_{X}^{*}\left(\alpha_{x}\right)=0$ if and only if $e \in A_{J(x)}$, for $x \in X$.

iii) Let $A^{\prime}$ be any complement of $A$ in $E$, so that $E=A \oplus A^{\prime}$. Let us identify $A^{\prime}$ with $\left\{\left((0,0),\left(0, a^{\prime}\right)\right) \mid a^{\prime} \in A^{\prime}\right\}$ in $\mathbb{T} X \times E$. Then $K \cap A^{\prime}=\{0\}$.

Proof. The first assertion in $i$ ) follows from $i$ ) and $i$ ) in Prop. 2.10. Note that $\rho_{X}: J^{*} A \rightarrow T X$ is defined by the inverse of (7) followed by projection on $T X$, so it is smooth, and it preserves brackets as a result of the integrability of $K$. The property $d J\left(\rho_{X}(a)\right)=\rho(a), a \in A_{J(x)}$, is a direct consequence of $\left.d 1\right)$ in Def. 2.4.

Consider the projection $p: K \rightarrow T^{*} X$. Using $\left.i i\right)$ in Prop. 2.10, one can deduce that $\operatorname{ker}(p)=K \cap(E \times(T X \oplus\{0\})) \cong A$. A dimension count shows that $p$ is surjective, so the first statement in $i$ ) follows. For the second assertion, we consider the pairing of $((u, \alpha), e) \in K$ with elements of the form $\left(\left(\rho_{X}(a), 0\right), a\right)$, for $a \in J^{*} A$ (which are necessarily in $K$ by definition of $\left.\rho_{X}\right)$, and use that $A$ is maximal isotropic.

Property iii) is a direct consequence of $i$ i) in Prop. 2.10 and $A \cap A^{\prime}=\{0\}$.

3.2. Two different characterizations. We now see how the intrinsic notion of Hamiltonian space for a Manin pair $(E, A)$, discussed in Section 3.1, has more familiar incarnations once extra (noncanonical) choices are made.

Quasi-Poisson geometry. Let $(E, A)$ be a Manin pair over $S$. The first possible noncanonical choice we consider is that of an isotropic splitting $j$ as in (3), defining a Lie quasi-bialgebroid structure on $A$ with cobracket $F_{j}: \Gamma(A) \rightarrow \wedge^{2} \Gamma(A)$, bundle map $\rho_{A^{*}}^{j}: A^{*} \rightarrow T M$ and 3-tensor $\chi_{j} \in \Gamma\left(\wedge^{3} A\right)$ defined as in (4).

Let $(X, J, K)$ be a Hamiltonian space for the Manin pair $(E, A)$. One can adapt the construction in [9] to show that, once $j$ is fixed, one naturally obtains a bivector field $\Pi_{X}^{j} \in \mathfrak{X}^{2}(X)$ as follows. Conditions $\left.i\right)$ and $\left.i i\right)$ in Prop. 2.10 imply that, for all $x \in X, K_{(x, J(x))} \cap\left((T X)_{x} \times\left(A^{*}\right)_{J(x)}\right)=\{0\}$, i.e.,

$$
\widehat{J}^{*} K \cap\left(T X \oplus J^{*} A^{*}\right)=\{0\}
$$

where $\widehat{J}=\operatorname{Id} \times J: X \rightarrow \operatorname{graph}(J) \subset X \times S$. Hence $\widehat{J}^{*} K$ is the graph of a skewsymmetric bundle map $T^{*} X \oplus J^{*} A \rightarrow T X \oplus J^{*} A^{*}$, determined by an element $\Lambda^{j} \in$ $\Gamma\left(\wedge^{2}\left(T X \oplus J^{*} A^{*}\right)\right)$. The bivector field $\Pi_{X}^{j}$ is the component of $\Lambda^{j}$ in $\Gamma\left(\wedge^{2} T X\right)$ : given $\alpha \in\left(T^{*} X\right)_{x}, i_{\alpha} \Pi_{X}^{j}$ is the only element in $(T X)_{x}$ satisfying

$$
\left(\left(i_{\alpha} \Pi_{X}^{j}, \alpha\right),\left(0,-\rho_{X}^{*} \alpha\right)\right) \in K_{(x, J(x))},
$$

where $\rho_{X}: J^{*} A \rightarrow T X$ is the action map given in Prop. 3.3, part $i$ ), see [9]. We have the following alternative characterization of $\Pi_{X}^{j}$ :

Lemma 3.4. Viewing $A^{*} \subset E$ via $j$, we have that $\operatorname{graph}\left(\Pi_{X}^{j}\right)=K \circ A^{*}$, where $\circ$ denotes composition of relations.

Proof. The composition of $K_{(x, J(x))}$ and $\left(A^{*}\right)_{J(x)}$ gives

$$
\left(K \circ A^{*}\right)_{x}=\left\{(u, \alpha) \in(\mathbb{T} X)_{x} \mid \exists e \in A_{J(x)}^{*} \text { with }((u, \alpha), e) \in K_{(x, J(x))}\right\} .
$$


This is a Lagrangian subspace of $\left(T X \oplus T^{*} X\right)_{x}$, so it suffices to show that graph $\left(\Pi_{X}^{j}\right) \subseteq$ $K \circ A^{*}$ pointwise. But this is a direct consequence of (8).

For an arbitrary Lie quasi-bialgebroid $\left(A, F, \chi, \rho_{A^{*}}\right)$ over $S$, there is a notion of Hamiltonian space (see e.g. [14]) defined by quadruples $\left(X, \Pi_{X}, J, \rho_{X}\right)$, where $X$ is a manifold, $\Pi_{X} \in \mathfrak{X}^{2}(X), J: X \rightarrow S$ is a smooth map (the moment map), and $\rho_{X}: J^{*} A \rightarrow T X$ is a Lie algebroid action, satisfying the following compatibility conditions:

$$
\begin{aligned}
& \frac{1}{2}\left[\Pi_{X}, \Pi_{X}\right]=\rho_{X}(\chi), \\
& \mathcal{L}_{\rho_{X}(a)} \Pi_{X}=\rho_{X}\left(d_{*} a\right), a \in \Gamma(A), \\
& \Pi_{X}^{\sharp} J^{*}=\rho_{X} \rho_{A^{*}}^{*},
\end{aligned}
$$

where $d_{*}$ is the quasi-differential on $\Gamma(\wedge A)$ determined by $F$ and $\rho_{A^{*}}$. These Hamiltonian spaces are also referred to as Hamiltonian quasi-Poisson spaces, and they form a category in which morphisms between $\left(X, \Pi_{X}, J, \rho_{X}\right)$ and $\left(X^{\prime}, \Pi_{X^{\prime}}, J^{\prime}, \rho_{X}^{\prime}\right)$ are smooth equivariant maps $f: X \rightarrow X^{\prime}$ such that $f_{*} \Pi_{X}=\Pi_{X^{\prime}}$ and $J^{\prime} \circ f=J$.

Given a Manin pair $(E, A)$ together with a splitting $j$, we denote by $\overline{\mathcal{M}}_{j}(E, A)$ the category of Hamiltonian spaces associated with the Lie quasi-bialgebroid $\left(A, F_{j}, \chi_{j}\right.$, $\left.\rho_{A^{*}}^{j}\right)$ defined by $j$. The following result generalizes $[9$, Thm. 4.1] and is proven similarly (c.f. [15]):

Proposition 3.5. Given a Hamiltonian space $(X, J, K)$ for $(E, A)$ and a splitting $j$, then $\left(X, \Pi_{X}^{j}, J, \rho_{X}\right)$ is a Hamiltonian space for the Lie quasi-bialgebroid determined by $j$; conversely, given a Hamiltonian space $\left(X, \Pi_{X}, J, \rho_{X}\right)$ for a Lie quasi-bialgebroid $\left(A, F, \chi, \rho_{A^{*}}\right)$, the bundle

$$
K:=\left\{\left(\left(\rho_{X}(a)+i_{\alpha} \Pi_{X}, \alpha\right),\left(a,-\rho_{X}^{*}(\alpha)\right)\right) \mid a \in J^{*} A, \alpha \in T^{*} X\right\}
$$

over graph $(J)$ makes $(X, J, K)$ into a Hamiltonian space for $(E, A)$, where $E=A \oplus A^{*}$ is the double Courant algebroid. Moreover, these constructions define an isomorphism of Hamiltonian categories $\overline{\mathcal{M}}(E, A) \cong \overline{\mathcal{M}}_{j}(E, A)$.

Example 3.6 (Quasi-Poisson $D / G$-valued moment maps). If $\left(E=\mathfrak{d}_{S}, A=\mathfrak{g}_{S}\right)$ is the Manin pair over $S=D / G$ of Example 2.2, and if $j$ is a splitting of the Manin pair $(\mathfrak{d}, \mathfrak{g})$ (i.e., $\mathfrak{h}=j\left(\mathfrak{g}^{*}\right)$ is an isotropic complement of $\mathfrak{g}$ in $\mathfrak{d}$ ), then the Hamiltonian spaces in $\overline{\mathcal{M}}_{j}(E, A)$ are exactly the Hamiltonian quasi-Poisson spaces with $D / G$ valued moment maps considered in [2] (c.f. [8, Sec. 5]). For the canonical Hamiltonian space $(S, \mathrm{Id}, K)$ of Example 3.2, the associated bivector field given by Prop. 3.5 agrees with the one in [2, Sec. 3.5] (c.f. [8, Sec. 5.1]); it is the bivector field determined by the Lie quasi-bialgebroid $\mathfrak{g}_{S}, \mathfrak{h}_{S} \subset \mathfrak{d}_{S}$ over $S$ (c.f. [14], [8, App. 4]) .

Dirac geometry. To define a second possible realization of the Hamiltonian category associated with a Manin pair $(E, A)$, we assume that $E$ is an exact Courant algebroid, i.e., that the sequence

$$
0 \rightarrow T^{*} S \stackrel{\rho^{*}}{\longrightarrow} E \stackrel{\rho}{\longrightarrow} T S \rightarrow 0
$$

is exact. One can always choose (in a noncanonical way) an isotropic splitting $s$ : $T S \rightarrow E$, which induces an identification of $E$ with $\mathbb{T} S=T S \oplus T^{*} S$, where the latter 
is equipped with the Courant algebroid structure of Example 2.3, with closed 3-form $\phi_{S}^{s} \in \Omega^{3}(S)$ defined by

$$
\phi_{S}^{s}\left(v_{1}, v_{2}, v_{3}\right):=\left\langle s\left(v_{1}\right), \llbracket s\left(v_{2}\right), s\left(v_{3}\right) \rrbracket\right\rangle .
$$

Under this identification, $A \subset E$ defines a Dirac structure $L_{S}^{s}$ on $S$ (with respect to $\left.\phi_{S}^{s}\right)$,

$$
L_{S}^{s}=\left\{\left(\rho(a), s^{*}(a)\right) \mid a \in A\right\} \subset \mathbb{T} S .
$$

We recall that there is a general notion of Hamiltonian space associated with any Dirac manifold $\left(S, L_{S}, \phi_{S}\right)[7,8]$, where we view $S$ as the target of the moment map. These Hamiltonian spaces are given by smooth maps $J: X \rightarrow S$, where $X$ is a manifold equipped with a Dirac structure $L_{X}$ for which $J$ is a strong Dirac map $[1,7,8]$; that is, $J: X \rightarrow S$ is a forward Dirac map [11], $L_{X}$ is integrable with respect to $J^{*} \phi_{S}$, and the following transversality condition holds: $\operatorname{ker}(d J) \cap\left(L_{X} \cap T X\right)=\{0\}$. We denote such Hamiltonian spaces by triples $\left(X, L_{X}, J\right)$. A morphism between Hamiltonian spaces $\left(X, L_{X}, J\right)$ and $\left(X^{\prime}, L_{X^{\prime}}, J^{\prime}\right)$ is a forward Dirac map $f: X \rightarrow X^{\prime}$ such that $J^{\prime} \circ f=J$, and the category of Hamiltonian spaces associated with $S$ is denoted by $\overline{\mathcal{M}}\left(S, L_{S}, \phi_{S}\right)$.

Given a Manin pair $(E, A)$ over a manifold $S$ such that $E$ is exact, and letting $s: T S \rightarrow E$ be an isotropic splitting of (13), we have the associated category of Hamiltonian spaces $\overline{\mathcal{M}}_{s}(E, A):=\overline{\mathcal{M}}\left(S, L_{S}^{s}, \phi_{S}^{s}\right)$. Let $\widehat{J}:=(\mathrm{Id}, J): X \rightarrow X \times S$, and consider the maps $\mathfrak{F}_{\widehat{J}}$ and $\mathfrak{B}_{\widehat{J}}$ from Prop. 2.8 (with $Q=X$, and $M=X \times S$ equipped with 3-form $0 \times \phi_{S}^{s}$ ).

Theorem 3.7. Given a Hamiltonian space $\left(X, L_{X}, J\right)$ for $\left(S, L_{S}^{s}, \phi_{S}^{s}\right)$, then $(X, J, K$ $=\mathfrak{F}_{\widehat{J}}\left(L_{X}\right)$ ) is a Hamiltonian space for $(E, A)$ and, given $(X, J, K)$ a Hamiltonian space for $(E, A)$, then $\left(X, L_{X}=\mathfrak{B}_{\widehat{J}}(K), J\right)$ is a Hamiltonian space for $\left(S, L_{S}^{s}, \phi_{S}^{s}\right)$. Moreover, these procedures establish an isomorphism of categories $\overline{\mathcal{M}}_{s}(E, A) \cong$ $\overline{\mathcal{M}}(E, A)$.

Proof. Given $\left(X, L_{X}, J\right)$, we consider

$$
K:=\mathfrak{F}_{\widehat{J}}\left(L_{X}\right)=\left\{\left(\left(u, \alpha-J^{*} \beta\right),(d J(u), \beta)\right) \mid(u, \alpha) \in L_{X}, \beta \in T^{*} S\right\},
$$

which is a Dirac structure in $\mathbb{T} X \times \mathbb{T} S$ supported on graph $(J)$ (integrable with respect to $\left.0 \times \phi_{S}^{s}\right)$. In order to show that $K$ defines a Hamiltonian space for the Manin pair $(E, A)=\left(\mathbb{T} S, L_{S}^{s}\right)$, we must show that it satisfies conditions $\left.i\right)$ and $\left.i i\right)$ in Prop. 2.10.

To prove $i)$, note that if $((u, 0),(0,0)) \in K$, then $(u, 0) \in L_{X} \cap T X$ and $u \in \operatorname{ker}(d J)$. Hence $u=0$ since $\operatorname{ker}(d J) \cap\left(L_{X} \cap T X\right)=\{0\}$. To prove $\left.i i\right)$, let $((u, 0),(d J(u), \beta)) \in$ $K$. Then $\left(u, J^{*} \beta\right) \in L_{X}$ and, since $J$ is forward Dirac map, we have $(d J(u), \beta) \in L_{S}$. On the other hand, if $(v, \beta) \in L_{S}$ then, using that $J:\left(X, L_{X}\right) \rightarrow\left(S, L_{S}\right)$ is a forward Dirac map, there exists $u \in T X$ such that $d J(u)=v$ and $\left(u, J^{*} \beta\right) \in L_{X}$. Thus, $((u, 0),(d J(u), \beta)) \in K$.

Let now $(X, J, K)$ be a Hamiltonian space for the Manin pair $(E, A)=\left(\mathbb{T} S, L_{S}^{s}\right)$. Then $L_{X}:=\mathfrak{B}_{\widehat{J}}(K)$ is a Dirac structure on $X$ (integrable with respect to $J^{*} \phi_{S}^{s}$ ). Explicitly,

$$
L_{X}=\left\{\left(u, \alpha+J^{*} \beta\right) \mid((u, \alpha),(d J(u), \beta)) \in K\right\} .
$$

To see that $J$ is a forward Dirac map, it suffices to show that $L_{S} \subseteq \mathfrak{F}_{J}\left(L_{X}\right)$ at each point $J(x)$. So take $(v, \beta) \in L_{S}$ at $J(x)$. By $\left.i i\right)$, there exists $u \in(T X)_{x}$ such 
that $((u, 0),(v, \beta)) \in K$, with $v=d J(u)$. It follows from (17) that $\left(u, J^{*} \beta\right) \in L_{X}$, so $(v, \beta) \in \mathfrak{F}_{J}\left(L_{X}\right)$. It remains to check that $\operatorname{ker}(d J) \cap\left(L_{X} \cap T X\right)=\{0\}$. From (17), we see that $u \in \operatorname{ker}(d J) \cap\left(L_{X} \cap T X\right)$ if and only if there exists $\beta \in T^{*} S$ with $\left(\left(u,-J^{*} \beta\right),(0, \beta)\right) \in K$. Now notice that any element of the form $\left(\left(0,-J^{*} \beta\right),(0, \beta)\right)$ is in $K$ (just check that the pairing of an element of this form with any element in $K$ must vanish using that $K$ is supported on the graph of $J$ ). It follows that $((u, 0),(0,0)) \in K$, hence $u=0$ by $i)$.

Finally, note that $f:\left(X, L_{X}\right) \rightarrow\left(X^{\prime}, L_{X^{\prime}}\right)$ is a forward Dirac map if and only if $L_{X^{\prime}}=\Gamma_{f} \circ L_{X}$, and, in case $J^{\prime} \circ f=J$, this is equivalent to $K^{\prime}=\Gamma_{f} \circ K$. So morphisms in $\overline{\mathcal{M}}(E, A)$ and $\overline{\mathcal{M}}_{s}(E, A)$ are naturally identified.

Example 3.8 (Dirac geometric $D / G$-valued moment maps). When $\left(E=\mathfrak{d}_{S}, A=\mathfrak{g}_{S}\right)$ is the Manin pair over $S=D / G$ of Example 2.2, and $s$ is an isotropic splitting of (13), then the Hamiltonian spaces in $\overline{\mathcal{M}}_{s}(E, A)$ coincide with the ones considered in [8, Sec. 4], and particular examples include the G-valued moment maps of [4] (c.f. [1, $7,8]$ ). For the canonical Hamiltonian space $(S, \mathrm{Id}, K)$ of Example 3.2, the associated Dirac structure on $S$ induced by $s$ is simply $L_{S}^{s}$ given in (15); the Dirac structure on a dressing $\mathfrak{g}$-orbit $\mathcal{O}$ is its presymplectic structure as a leaf of the Dirac manifold $\left(S, L_{S}^{s}\right)$.

3.3. Equivalence. Let $(E, A)$ be a Manin pair over a manifold $S$, and assume that $E$ is an exact Courant algebroid. Let us fix both types of extra choices considered in Section 3.2: an isotropic splitting $j: A^{*} \rightarrow E$ (making $A$ into a Lie quasi-algebroid $\left(A, F, \chi, \rho_{A^{*}}\right)$ given by (4)) as well as an identification $E \cong T S \oplus T^{*} S$, induced by $s: T S \rightarrow E$, where the Courant algebroid structure on $\mathbb{T} S$ is with respect to the 3form $\phi_{S}$ given in (14). (We are simplifying the notations by omitting the dependence on $j$ and s.) Under this identification, $A \subset E$ defines a Dirac structure $L_{S}$ on $S$ (15), whereas the subbundle $A^{*} \subset E$ (via $j$ ) defines a transverse almost Dirac structure $C_{S}$. As discussed in Section 3.2, each choice leads to a category of Hamiltonian spaces, denoted by $\overline{\mathcal{M}}_{j}(E, A)$ and $\overline{\mathcal{M}}_{s}(E, A)$. Since Prop. 3.5 and Thm. 3.7 say that both categories are different realizations of the same category $\overline{\mathcal{M}}(E, A)$, we immediately obtain:

Theorem 3.9. There is an isomorphism of categories $\overline{\mathcal{M}}_{j}(E, A) \cong \overline{\mathcal{M}}_{s}(E, A)$ as follows: given a Hamiltonian space $\left(X, \Pi_{X}, J, \rho_{X}\right)$ for the Lie quasi-bialgebroid $(A, F, \chi$, $\left.\rho_{A^{*}}\right)$, then the triple $\left(X, L_{X}, J\right)$ is a Hamiltonian space for the Dirac manifold $\left(S, L_{S}\right.$, $\left.\phi_{S}\right)$, where

$$
L_{X}:=\left\{\left(\rho_{X}(a)+i_{\alpha} \Pi_{X}, J^{*} s^{*}(a)+\left(\operatorname{Id}-J^{*} \bar{\rho}^{*} \rho_{X}^{*}\right) \alpha\right) \mid a \in A, \alpha \in T^{*} X\right\},
$$

with $\bar{\rho}=j^{*} s: T S \rightarrow A$. Conversely, given a Hamiltonian space $\left(X, L_{X}, J\right)$ for $\left(S, L_{S}, \phi_{S}\right)$, then the composition of relations $\mathfrak{F}_{\widehat{J}}\left(L_{X}\right) \circ A^{*} \subset \mathbb{T} X$ defines a bivector field $\Pi_{X} \in \mathfrak{X}^{2}(X)$,

$$
\operatorname{graph}\left(\Pi_{X}\right)=\mathfrak{F}_{\widehat{J}}\left(L_{X}\right) \circ A^{*},
$$

making $\left(X, \Pi_{X}, J, \rho_{X}\right)$ into a Hamiltonian quasi-Poisson space for $\left(A, F, \chi, \rho_{A^{*}}\right)$.

Proof. The proof follows by combining the constructions identifying both categories $\overline{\mathcal{M}}_{j}(E, A)$ and $\overline{\mathcal{M}}_{s}(E, A)$ with $\overline{\mathcal{M}}(E, A)$ : the expression for the Dirac structure (18) 
is obtained directly as $\mathfrak{B}_{\widehat{J}}(K)$, where $K$ is given by (12). Conversely, the expression for the quasi-Poisson bivector field (19) follows from Lemma 3.4.

Let us denote by $\mathcal{M}(E, A)$ the subcategory of $\overline{\mathcal{M}}(E, A)$ consisting of Hamiltonian spaces satisfying the additional condition that the natural projection of $K$ on $T X$ is onto. Then the isomorphism in Thm. 3.9 restricts to an isomorphism of subcategories,

$$
\mathcal{M}_{j}(E, A) \cong \mathcal{M}(E, A) \cong \mathcal{M}_{s}(E, A),
$$

where Hamiltonian spaces $\left(X, \Pi_{X}, J, \rho_{X}\right)$ in $\mathcal{M}_{j}(E, A)$ satisfy the extra condition $T X=\left\{\rho_{X}(a)+i_{\alpha} \Pi_{X} \mid a \in J^{*} A, \alpha \in T^{*} X\right\}$, and Hamiltonian spaces $\left(X, L_{X}, J\right)$ in $\mathcal{M}_{s}(E, A)$ are such that $L_{X}=\operatorname{graph}\left(\omega_{X}\right)$ for a given 2 -form $\omega_{X} \in \Omega^{2}(X)$.

Remark 3.10. The bivector field $\Pi_{X}$ (19) admits another description, following $[1$, 8]. Using (16), the expression in (19) can be written explicitly as

$$
\operatorname{graph}\left(\Pi_{X}\right)_{x}=\left\{\left(u, \alpha-J^{*} \beta\right) \in(\mathbb{T} X)_{x} \mid(d J(u), \beta) \in C_{S},(u, \alpha) \in L_{X}\right\} .
$$

On the other hand, as shown in [1], the pull-back image (in the Dirac geometric sense [11]) of $C_{S}$ under $J$ defines an almost Dirac structure $C_{X}$ on $X$ transverse to $L_{X}$. The splitting $\mathbb{T} X=L_{X} \oplus C_{X}$ defines a Lie quasi-bialgebroid, and it naturally induces a bivector field $\Pi$ on $X$ (see e.g. [14] or [8, App. 4]). By [1, Prop. 1.16], such $\Pi$ is given by

$$
\operatorname{graph}(\Pi)_{x}=\left\{\left(u, \alpha-J^{*} \beta\right) \in(\mathbb{T} X)_{x} \mid\left(u, J^{*} \beta\right) \in C_{X},(u, \alpha) \in L_{X}\right\} .
$$

Since $C_{X}$ is the pull-back of $C_{S}$, it immediately follows that $\operatorname{graph}\left(\Pi_{X}\right) \subseteq \operatorname{graph}(\Pi)$. This implies that $\Pi_{X}=\Pi$.

Example 3.11. For the particular Manin pair $\left(E=\mathfrak{d}_{S}, A=\mathfrak{g}_{S}\right)$ over $S=D / G$ of Example 2.2, Thm. 3.9 recovers the equivalence proven in [8, Sec. 6.3]; this result gives, as a special case, the equivalence between the two formulations of G-valued moment maps in [4] and [3] (see [3, Sec. 10], [1, Sec. 5.4], [7, Sec. 3.5] for proofs).

3.4. Poisson algebras and moment map reduction. Let $(X, J, K)$ be a Hamiltonian space for a Manin pair $(E, A)$ over $S$. Following Prop. 3.3, part $i$ ), let $\rho_{X}$ denote the induced Lie algebroid action of $A$ on $J: X \rightarrow S$.

A function $f \in C^{\infty}(X)$ is called admissible if there exists a vector field $u_{f} \in \mathfrak{X}(X)$ satisfying

$$
\left(\left(u_{f}, d f\right)_{x}, 0\right) \in K_{(x, J(x))}, \quad \forall x \in X .
$$

By $i$ ) in Prop. 2.10, $u_{f}$ is uniquely defined by condition (20). Since $K$ is supported on $\operatorname{graph}(J), u_{f}$ satisfies $d J\left(u_{f}\right)=0$. It is simple to check that the set of admissible functions $C^{\infty}(X)_{\text {adm }}$ is a subalgebra of $C^{\infty}(X)$. We define a bracket on $C^{\infty}(X)_{\text {adm }}$ by

$$
\{f, g\}:=\mathcal{L}_{u_{f}} g .
$$

Lemma 3.12. $\left(C^{\infty}(X)_{\mathrm{adm}},\{\cdot, \cdot\}\right)$ is a Poisson algebra.

Proof. Let $f$ and $g$ be admissible functions. The fact that $K$ is isotropic implies that $d f\left(u_{g}\right)=-d g\left(u_{f}\right)$, i.e., $\{\cdot, \cdot\}$ is skew-symmetric. Using the integrability of $K$ with respect to the product Courant bracket on $\mathbb{T} X \times E$ (condition $d 2$ ) in Def. 2.4), one can check that $\{f, g\}$ is admissible; in fact, $u_{\{f, g\}}=\left[u_{f}, u_{g}\right]$. This last property also proves the Jacobi identity for $\{\cdot, \cdot\}$. 
Lemma 3.13. A function $f$ satisfies $\mathcal{L}_{\rho_{X}(a)} f=0$ for all $a \in A_{J(x)}$ if and only if there exists $u_{x} \in(T X)_{x}$ such that $\left(\left(u_{x},(d f)_{x}\right), 0\right) \in K_{(x, J(x))}$.

Proof. Since $\left(\left(\rho_{X}(a), 0\right), a\right) \in K$, if we assume that $\left(\left(u_{x},(d f)_{x}\right), 0\right) \in K_{(x, J(x))}$ and use that $K$ is isotropic, it follows that $d f\left(\rho_{X}(a)\right)=\mathcal{L}_{\rho_{X}(a)} f=0$. Conversely, suppose that $\mathcal{L}_{\rho_{X}(a)} f=0, a \in A_{J(x)}$. From Prop. 3.3, part ii), there exists $u^{\prime} \in T_{x} X$, $a^{\prime} \in A_{J(x)}$ such that $\left(\left(u^{\prime}, d f\right), a^{\prime}\right) \in K$. Since $\left(\left(\rho_{X}\left(a^{\prime}\right), 0\right), a^{\prime}\right) \in K_{(x, J(x))}$, we have that $\left(\left(u_{x},(d f)_{x}\right), 0\right) \in K_{(x, J(x))}$, for $u_{x}=u^{\prime}-\rho_{X}\left(a^{\prime}\right)$.

A function $f \in C^{\infty}(X)$ is called $A$-invariant if $\mathcal{L}_{\rho_{X}(a)} f=0, \forall a \in \Gamma(A)$, and the set of all $A$-invariant functions is denoted by $C^{\infty}(X)^{A}$.

Proposition 3.14. A function $f \in C^{\infty}(X)$ is A-invariant if and only if it is admissible, and therefore $C^{\infty}(X)^{A}$ is a Poisson algebra.

Proof. From Lemma 3.13, we know that $f$ is $A$-invariant if and only if, at each $x \in X$, there exists $u_{x} \in(T X)_{x}$ such that $\left(\left(u_{x},(d f)_{x}\right), 0\right) \in K_{(x, J(x))}$. It remains to check that $u_{f}: X \rightarrow T X, x \mapsto u_{x}$, is a smooth vector field. To see that, fix any vector subbundle $A^{\prime} \subset E$ such that $E=A \oplus A^{\prime}$, which defines a projection $p: K \rightarrow T^{*} X \oplus J^{*} A$; note that $p$ is injective since $K \cap\left((T X \oplus\{0\}) \times A^{\prime}\right)=\{0\}$ by ii) in Prop. 2.10, and it is onto by dimension count. Now $u_{f}$ is defined by $p^{-1}(d f)$ followed by the projection $K \rightarrow T X$, so it is smooth. Hence $C^{\infty}(X)^{A}$ agrees with the algebra of admissible functions, so it is a Poisson algebra by Lemma 3.12.

Remark 3.15. We can fix an isotropic splitting $j: A^{*} \rightarrow E$, and let $\Pi_{X}^{j}$ be the associated quasi-Poisson bivector field on $X$. The vector field associated with $f \in$ $C^{\infty}(X)^{A}$, constructed in Prop. 3.14, is $u_{f}=i_{d f} \Pi_{X}^{j}$ (it is independent of $j$ ). The canonical Poisson structure on $C^{\infty}(X)^{A}$ acquires a concrete expression in terms of $\Pi_{X}^{j}$ : for $f, g \in C^{\infty}(X)^{A},\{f, g\}=\Pi_{X}^{j}(d f, d g)$. On the other hand, when $E$ is exact and a splitting $s$ is fixed, $X$ inherits a Dirac structure $L_{X}^{s}$, and $C^{\infty}(X)^{A}$ is a Poisson subalgebra of its algebra of admissible functions (in the sense of [13]), characterized by functions $f$ admitting a Hamiltonian vector field $u_{f}$ satisfying $d J\left(u_{f}\right)=0$.

One can also perform moment map reduction for Hamiltonian spaces for Manin pairs $(E, A)$ :

Proposition 3.16. Let $(X, J, K)$ be a Hamiltonian space for a Manin pair $(E, A)$ over $S$. Let $\mathcal{O} \subset S$ be an orbit of $A$ (i.e., an integral leaf of the distribution $\rho(A) \subseteq T S$ ) such that $J: X \rightarrow S$ is transverse to $\mathcal{O}$. Then the $A$-action on $X$ is tangent to the submanifold $J^{-1}(\mathcal{O}) \subset X$, and the space $C^{\infty}\left(J^{-1}(\mathcal{O})\right)^{A}$ of $A$ invariant functions on $J^{-1}(\mathcal{O})$ inherits a Poisson bracket for which the restriction $C^{\infty}(X)^{A} \rightarrow C^{\infty}\left(J^{-1}(\mathcal{O})\right)^{A}$ is a Poisson map.

Proof. From Prop. 3.3, part $i)$, we know that $d J\left(\rho_{X}(a)\right)=\rho(a)$ for all $a \in J^{*} A$. This shows that the $A$-action is tangent to $J^{-1}(\mathcal{O})$. Take $f \in C^{\infty}\left(J^{-1}(\mathcal{O})\right)^{A}$, and let $\tilde{f}$ be an arbitrary extension of $f$ to $X$. We claim that there exists $u_{\tilde{f}} \in \mathfrak{X}\left(J^{-1}(\mathcal{O})\right)$ satisfying the condition

$$
\left(\left(u_{\tilde{f}}, d \widetilde{f}\right)_{x}, 0\right) \in K, \quad x \in J^{-1}(\mathcal{O}) .
$$

(The condition determines $u_{\tilde{f}}$ uniquely by $i$ ) in Prop. 2.10.) Indeed, recall from the proof of Prop. 3.14 that the projection $K \rightarrow T^{*} X \oplus J^{*} A$ induced by the choice 
of a complement $A^{\prime}$ of $A$ in $E$ is an isomorphism. So one can find $\widetilde{u}_{\widetilde{f}} \in \mathfrak{X}(X)$ and $a^{\prime} \in \Gamma\left(J^{*} A^{\prime}\right)$ such that $\left(\left(\widetilde{u}_{\tilde{f}}, d \widetilde{f}\right), a^{\prime}\right) \in K$. Since $f$ is $A$-invariant, we have $\left.a^{\prime}\right|_{J^{-1}(\mathcal{O})}=0$ (by Lemma 3.13 and $i i$ ) in Prop. 2.10), which implies that $d J\left(\widetilde{u}_{\tilde{f}}\right)=0$ over $J^{-1}(\mathcal{O})$. Hence $u_{\tilde{f}}:=\left.\widetilde{u}_{\tilde{f}}\right|_{J^{-1}(\mathcal{O})}$ is tangent to $J^{-1}(\mathcal{O})$ and satisfies (22).

Given $g \in C^{\infty}\left(J^{-1}(\mathcal{O})\right)^{A}$, with extension $\widetilde{g}$ to $X$, then $K$ being isotropic implies that $\mathcal{L}_{u_{\tilde{f}}} g=-\mathcal{L}_{u_{\tilde{g}}} f$. As a result, the bracket $\{f, g\}:=\mathcal{L}_{u_{\tilde{f}}} g=-\mathcal{L}_{u_{\tilde{g}}} f$ is well-defined, i.e., it only depends on $f, g$, and not on their extensions. To check the Jacobi identity, we use $d 2$ ) in Def. 2.4. For a given extension $\widetilde{f}$ of $f \in C^{\infty}\left(J^{-1}(\mathcal{O})\right)^{A}$, we can always find a section $e \in \Gamma(\{0\} \times E)=C^{\infty}(X \times S, E)$ such that $\xi_{\tilde{f}} \in \Gamma(\mathbb{T} X \times E)$, given at $(x, y) \in X \times S$ by $\left(\left(\widetilde{u}_{\tilde{f}}, d \widetilde{f}\right)_{x}, e_{(x, y)}\right)$, satisfies $\left.\left(\xi_{\widetilde{f}}\right)\right|_{\operatorname{graph}(J)} \in K$ and $e_{(x, J(x))}=0$ if $x \in J^{-1}(\mathcal{O})$. From Remark 2.5, we see that the restriction of $\llbracket \xi_{\tilde{f}}, \xi_{\widetilde{g}} \rrbracket$ to the submanifold $\left\{(x, J(x)), x \in J^{-1}(\mathcal{O})\right\}$ gives $\left(\left(\left[u_{\tilde{f}}, u_{\tilde{g}}\right], \mathcal{L}_{u_{\tilde{f}}} g\right), 0\right) \in K$, which implies the Jacobi identity. Hence the algebra $C^{\infty}\left(J^{-1}(\mathcal{O})\right)^{A}$ has a canonical Poisson bracket, and it is clear from the construction that the restriction $C^{\infty}(X)^{A} \rightarrow C^{\infty}\left(J^{-1}(\mathcal{O})\right)^{A}$ is a Poisson map.

Remark 3.17. When an isotropic splitting $j$ of $E$ is fixed, then the vector field $u_{\widetilde{f}}$ in the proof Prop. 3.16 is given by $i_{d \widetilde{f}} \Pi_{X}^{j}$, and the Poisson bracket on $C^{\infty}\left(J^{-1}(\mathcal{O})\right)^{A}$ can be computed by $\{f, g\}:=\left.\Pi_{X}^{j}(d \widetilde{f}, d \widetilde{g})\right|_{J^{-1}(\mathcal{O})}$ (one can also directly verify that this is independent of extensions and that it is a Poisson bracket by (9) and (10)). If $E$ is an exact Courant algebroid, one can alternatively describe the Poisson algebra $C^{\infty}\left(J^{-1}(\mathcal{O})\right)^{A}$ via Dirac reduction, by identifying this algebra with the admissible functions of the pull-back image of $L_{X}^{s}$ to $J^{-1}(\mathcal{O})$, see [7, Sec. 4.4] (c.f [8, Sec. 6.4]).

\section{Examples from Lie groupoids}

In this section, we consider examples of Manin pairs and Hamiltonian spaces arising from the theory of Lie groupoids. Specifically, we let $(E, A)$ be a Manin pair over $S$, and take $\mathcal{G} \rightrightarrows S$ to be the source-simply connected Lie groupoid integrating $A$, viewed as a Lie algebroid (which we assume to be integrable). This groupoid may acquire different geometrical structures via integration: on the one hand, the choice of a splitting $j$ as in (3) determines a bivector field $\Pi \in \mathfrak{X}^{2}(\mathcal{G})$ making $\mathcal{G}$ a quasi-Poisson groupoid [14] (integrating the Lie quasi-bialgebroid (4)); on the other hand, if $E$ is exact and $s$ is a splitting of (13), then $A$ is identified with a Dirac structure $L_{S} \subset \mathbb{T} S$ which, according to [10], integrates to a 2-form $\omega \in \Omega^{2}(\mathcal{G})$ making $\mathcal{G}$ a presymplectic groupoid. The goal of this section is to establish a direct relationship between the two types of integration, $\Pi$ and $\omega$, in the light of Thm. 3.9.

4.1. Multiplicative Dirac structures. Let $\mathcal{H} \rightrightarrows \mathcal{H}_{0}$ be a Lie groupoid, and let $\mathcal{G}$ be an embedded subgroupoid of $\mathcal{H}$, with inclusion homomorphism $f: \mathcal{G} \hookrightarrow \mathcal{H}$ (over $\left.f_{0}: \mathcal{G}_{0} \hookrightarrow \mathcal{H}_{0}\right)$. We consider the associated tangent and cotangent Lie groupoids $T \mathcal{H} \rightrightarrows T \mathcal{H}_{0}$ and $T^{*} \mathcal{H} \rightrightarrows A^{*}(\mathcal{H})$, see e.g. [19]. We observe that there are also natural Lie groupoid structures on the pull-back bundles $f^{*} T \mathcal{H}, f^{*} T^{*} \mathcal{H}$, and on the direct sums $\mathbb{T} \mathcal{H}=T \mathcal{H} \oplus T^{*} \mathcal{H}, f^{*} T \mathcal{H} \oplus f^{*} T^{*} \mathcal{H}$, and $f^{*} T \mathcal{H} \oplus T^{*} \mathcal{G}$.

An (almost) Dirac structure $L$ on $\mathcal{H}$ supported on $f(\mathcal{G})$ is called multiplicative if $L \subset f^{*} \mathbb{T} \mathcal{H}$ is a subgroupoid, i.e., closed under multiplication and inversion. For $\mathcal{G}=$ 
$\mathcal{H}$, this unifies the usual notions of multiplicative bivector field (when $L=\operatorname{graph}(\Pi)$, for $\Pi \in \mathfrak{X}^{2}(\mathcal{G})$ ) and multiplicative 2-form (when $L=\operatorname{graph}(\omega)$, for $\omega \in \Omega^{2}(\mathcal{G})$ ), see e.g. [19].

Let us consider the map $\mathfrak{F}_{f}: \operatorname{Dir}(\mathcal{G}) \rightarrow \operatorname{Dir}(\mathcal{H})_{f(\mathcal{G})}$ as defined in Prop. 2.8 (however, in the present situation, we may consider just almost Dirac structures).

Lemma 4.1. If $L \subset \mathbb{T} \mathcal{G}$ is a multiplicative Dirac structure, then $\mathfrak{F}_{f}(L) \subset f^{*} \mathbb{T} \mathcal{H}$ is a multiplicative Dirac structure supported on $f(\mathcal{G})$.

Proof. As in the proof of Prop. 2.8, we consider the maps $\psi: \mathbb{T} \mathcal{G} \rightarrow f^{*} T \mathcal{H} \oplus T^{*} \mathcal{G}$, $\psi(u, \alpha)=(d f(u), \alpha)$, and $p: f^{*} \mathbb{T} \mathcal{H} \rightarrow f^{*} T \mathcal{H} \oplus T^{*} \mathcal{G}, p(v, \beta)=\left(v, f^{*} \beta\right)$. Since the maps $d f: T \mathcal{G} \rightarrow f^{*} T \mathcal{H}$ and $d f^{*}: f^{*} T^{*} \mathcal{H} \rightarrow T^{*} \mathcal{G}$ are groupoid morphisms, so are $\psi$ and $p$. Hence $\mathfrak{F}_{f}(L)=p^{-1}(\psi(L)) \subset f^{*} \mathbb{T} \mathcal{H}$ is a subgroupoid.

Let us consider Lie groupoids $\mathcal{G} \rightrightarrows \mathcal{G}_{0}, \mathcal{R} \rightrightarrows \mathcal{R}_{0}$, and a homomorphism $J: \mathcal{G} \rightarrow \mathcal{R}$. Then $f: \mathcal{G} \hookrightarrow \mathcal{G} \times \mathcal{R}, g \mapsto(g, J(g))$ defines an embedded Lie subgroupoid. Let $K$ be a multiplicative (almost) Dirac structure on $\mathcal{H}:=\mathcal{G} \times \mathcal{R}$ supported on $f(\mathcal{G})=\operatorname{graph}(J)$, i.e., $K \subset \mathbb{T} \mathcal{G} \oplus J^{*} \mathbb{T} \mathcal{R}$ is a subgroupoid. Let $C_{\mathcal{R}} \subset \mathbb{T} \mathcal{R}$ be a multiplicative almost Dirac structure on $\mathcal{R}$.

Lemma 4.2. Assume that $K$ and $C_{\mathcal{R}}$ satisfy the transversality condition $\left(0 \oplus J^{*} C_{\mathcal{R}}\right) \cap$ $K=\{0\}$ in $\mathbb{T} \mathcal{G} \oplus J^{*} \mathbb{T} \mathcal{R}$. Then the composition $K \circ C_{\mathcal{R}}$ is a multiplicative almost Dirac structure on $\mathcal{G}$.

Proof. Let $\operatorname{pr}_{\mathcal{G}}: \mathbb{T} \mathcal{G} \oplus J^{*} \mathbb{T} \mathcal{R} \rightarrow \mathbb{T} \mathcal{G}$ denote the natural projection. The composition $K \circ C_{\mathcal{R}}$,

$$
\left(K \circ C_{\mathcal{R}}\right)_{g}:=\left\{(u, \alpha) \in(\mathbb{T} \mathcal{G})_{g} \mid \exists(v, \beta) \in\left(C_{\mathcal{R}}\right)_{J(g)} \text { s.t. }((u, \alpha),(v, \beta)) \in K_{(g, J(g))}\right\},
$$

$g \in \mathcal{G}$, can be written as $\operatorname{pr}_{\mathcal{G}}\left(\left(\mathbb{T} \mathcal{G} \oplus J^{*} C_{\mathcal{R}}\right) \cap K\right)$. Since both $\mathbb{T} \mathcal{G} \oplus J^{*} C_{\mathcal{R}}$ and $K$ are subgroupoids of $\mathbb{T} \mathcal{G} \oplus J^{*} \mathbb{T} \mathcal{R}$, so is the intersection $\left(\mathbb{T} \mathcal{G} \oplus J^{*} C_{\mathcal{R}}\right) \cap K$. The transversality condition $\left(0 \oplus J^{*} C_{\mathcal{R}}\right) \cap K=\{0\}$ in $\mathbb{T} \mathcal{G} \oplus J^{*} \mathbb{T} \mathcal{R}$ says that the restriction of $\operatorname{pr}_{\mathcal{G}}$ to $\left(\mathbb{T} \mathcal{G} \oplus J^{*} C_{\mathcal{R}}\right) \cap K$ is an isomorphism onto $K \circ C_{\mathcal{R}}$. Since $\operatorname{dim}\left(\left(K \circ C_{\mathcal{R}}\right)_{g}\right)=\operatorname{dim}(\mathcal{G})$ at all $g$, it follows that $\left(\mathbb{T} \mathcal{G} \oplus J^{*} C_{\mathcal{R}}\right) \cap K$ has constant rank, so it is a smooth vector bundle, and hence $K \circ C_{\mathcal{R}}$ is a smooth vector bundle. Since $\operatorname{pr}_{\mathcal{G}}$ is a groupoid morphism, $K \circ C_{\mathcal{R}}$ is a subgroupoid of $\mathbb{T} \mathcal{G}$.

4.2. Presymplectic and quasi-Poisson groupoids. We now resume the discussion about presymplectic and quasi-Poisson groupoids. Our set-up is a Manin pair over $S$ for which the Courant algebroid is exact. We fix splittings $j$ and $s$ as in Section 3.2 , so that, after identifications, we have the following situation: $S$ is equipped with a Dirac structure $L_{S}$ (integrable with respect to $\phi_{S}$ ), $C_{S}$ is an almost Dirac structure such that $\mathbb{T} S=L_{S} \oplus C_{S}$. Let us assume that $L_{S}$, viewed as a Lie algebroid, admits an integration to a Lie groupoid, and let $\mathcal{G} \rightrightarrows S$ be the source-simply-connected Lie groupoid integrating $L_{S}$, with source/target maps denoted by $\mathbf{s}, \mathbf{t}$, and inversion $i: \mathcal{G} \rightarrow \mathcal{G}$. We know from [10] that, since $L_{S} \subset \mathbb{T} S$ is a Dirac structure, $\mathcal{G}$ has a 2 -form $\omega$ making it into a presymplectic groupoid; on the other hand, since $L_{S}, C_{S}$ define a Lie quasi-biagebroid, $\mathcal{G}$ inherits a bivector field $\Pi$ making it a quasi-Poisson groupoid [14].

It results from $(\mathcal{G}, \omega)$ being a presymplectic groupoid that $J=(\mathbf{t}, \mathbf{s}): \mathcal{G} \rightarrow S \times$ $S^{\text {op }}$ is a strong Dirac map [10, 27], where $S^{\text {op }}$ is equipped with the Dirac structure 
$L_{S}^{\mathrm{op}}:=\left\{(v, \beta) \mid(v,-\beta) \in L_{S}\right\}$ (integrable with respect to $\left.-\phi_{S}\right)$. In other words, $(\mathcal{G}, \operatorname{graph}(\omega), J)$ is a Hamiltonian space for the Dirac manifold $S \times S^{\text {op }}$. The induced action $\rho_{\mathcal{G}}$ of $A=L_{S} \times L_{S}^{\text {op }}$ on $J: \mathcal{G} \rightarrow S \times S^{\text {op }}$ is

$$
\rho_{\mathcal{G}}(u, v)=r_{g}(u)-l_{g}(v), \quad u \in\left(L_{S}\right)_{\mathbf{t}(g)}, v \in\left(L_{S}^{\mathrm{op}}\right)_{\mathbf{s}(g)},
$$

where $r_{g}$ and $l_{g}$ denote right/left translations on $\mathcal{G}$ (as well as their tangent maps).

By Thm. 3.9, there is a bivector field $\Pi_{\mathcal{G}} \in \mathfrak{X}^{2}(\mathcal{G})$ corresponding to $\omega$, and making $\mathcal{G}$ into a Hamiltonian quasi-Poisson space for the Lie quasi-bialgebroid determined by the pair $A=L_{S} \times L_{S}^{\mathrm{op}}, A^{*} \cong C_{S} \oplus C_{S}^{\mathrm{op}}$ (with action (23) and moment map $J$ ).

Theorem 4.3. The quasi-Poisson bivector field $\Pi_{\mathcal{G}}$ corresponding to $\omega$ via Thm. 3.9 agrees with the multiplicative bivector field $\Pi$ integrating the Lie quasi-bialgebroid determined by the splitting $\mathbb{T} S=L_{S} \oplus C_{S}$.

Proof. We denote by $\chi \in \Gamma\left(\wedge^{3} L_{S}\right)$ the 3-tensor and by $d_{*}$ the quasi-differential on $\Gamma\left(\wedge L_{S}\right)$ defined by the Lie quasi-bialgebroid $\mathbb{T} S=L_{S} \oplus C_{S}$. According to [14, Thm. 2.34], in order to prove that $\Pi_{\mathcal{G}}=\Pi$, one must check that $\Pi_{\mathcal{G}}$ is multiplicative, and that it satisfies

$$
\left(d_{*} f\right)^{r}=-\left[\Pi_{\mathcal{G}}, \mathbf{t}^{*} f\right], \quad \text { and } \quad\left(d_{*} a\right)^{r}=-\left[\Pi_{\mathcal{G}}, a^{r}\right],
$$

for all $f \in C^{\infty}(S)$ and $a \in \Gamma\left(L_{S}\right)$ (for $\xi \in \wedge^{k}\left(L_{S}\right), \xi^{r}$ is defined by $\left(\xi^{r}\right)_{g}=r_{g}\left(\xi_{\mathbf{t}(g)}\right)$, $g \in \mathcal{G})$.

To show that $\Pi_{\mathcal{G}}$ is multiplicative, note first that $J=(\mathbf{t}, \mathbf{s}): \mathcal{G} \rightarrow S \times S$ is a groupoid morphism, where $\mathcal{R}=S \times S$ is viewed as the pair groupoid. Hence $\widehat{J}=(\operatorname{Id}, J): \mathcal{G} \rightarrow \mathcal{H}$, where $\mathcal{H}:=\mathcal{G} \times \mathcal{R}$, is an embedding which is a groupoid morphism. If follows from Lemma 4.1 that $K:=\mathfrak{F}_{\widehat{J}}\left(L_{\mathcal{G}}\right)$ is a multiplicative Dirac structure in $\mathbb{T} \mathcal{H}=\mathbb{T} \mathcal{G} \times \mathbb{T} \mathcal{R}$ supported on graph $(J)$. Taking $C_{\mathcal{R}}=C_{S} \times C_{S}^{\text {op }}$ (writing out the groupoid structure on $\mathbb{T} \mathcal{R}$, for $\mathcal{R}=S \times S$ the pair groupoid, one directly sees that $C_{\mathcal{R}}$ is a multiplicative almost Dirac structure), it follows from Prop. 3.3, part iii), and Lemma 4.2 that the composition $K \circ C_{\mathcal{R}} \subset \mathbb{T} \mathcal{G}$ is multiplicative. This implies that $\Pi_{\mathcal{G}}$ is multiplicative by (19). On the other hand, the conditions in (24) follow directly from (10) and (11) (using the action (23)).

One can also verify, using $(9)$, that $\frac{1}{2}\left[\Pi_{\mathcal{G}}, \Pi_{\mathcal{G}}\right]=\chi^{r}-\chi^{l}$, where $\left(\chi^{l}\right)_{g}=l_{g}\left(i\left(\chi_{\mathbf{s}(g)}\right)\right)$, in accordance with [14].

Thm. 3.9 provides an explicit construction going from presymplectic to quasiPoisson groupoids (and vice-versa), thereby relating the two integration problems. In the particular case of a twisted symplectic groupoid (i.e., when $\omega$ is nondegenerate), the Dirac structure $L_{S}$ must be the graph of a bivector field [12] (i.e., it is a twisted Poisson structure in the sense of [26]), so we can take $C_{S}$ to be $T S$. In this case, the quasi-Poisson bivector field constructed in Thm. 3.9 is just the inverse of $\omega$, and Thm. 4.3 recovers [14, Prop. 4.5].

\section{Acknowledgments}

We thank P. Xu for stimulating discussions and Zhuo Chen and the referee for their comments. We acknowledge the financial support of CNPq (H.B.), MEC Research Contract "Juan de la Cierva", grant MTM2007-62478 (D.I.P.) and the Swiss National Science Fundation (P.Š.). We also thank several institutions for hosting us while this 
work was being done, including IMPA (D.I.P.), University of La Laguna (H.B. and D.I.P.) and the Erwin Schrödinger Institute.

\section{References}

[1] A. Alekseev, H. Bursztyn and E. Meinrenken, Pure spinors on Lie groups, arXiv:0709.1452 [Math.DG].

[2] A. Alekseev and Y. Kosmann-Schwarzbach, Manin Pairs and moment maps, J. Differential Geom. 56 (2000) 133-165.

[3] A. Alekseev, Y. Kosmann-Schwarzbach and E. Meinrenken, Quasi-Poisson manifolds, Canad. J. Math. 54 (2002) 3-29.

[4] A. Alekseev, A. Malkin and E. Meinrenken, Lie group valued moment maps, J. Differential Geom. 48 (1998), 445-495.

[5] A. Alekseev and P. Xu, Derived brackets and Courant algebroids, unpublished manuscript.

[6] H. Bursztyn, A. Cattaneo, R. Mehta, M. Zambon, in preparation.

[7] H. Bursztyn and M. Crainic, Dirac structures, momentum maps and quasi-Poisson manifolds, The breadth of symplectic and Poisson geometry, 1-40, Progr. Math. 232, Birkhäuser Boston, 2005.

[8] Dirac geometry, quasi-Poisson actions, and $D / G$-valued moment maps, Arxiv:0710.0639 [Math.DG].

[9] H. Bursztyn, M. Crainic and P. Ševera, Quasi-Poisson structures as Dirac structures, Travaux Mathématiques, XVI (2005), 41-52.

[10] H. Bursztyn, M. Crainic, A. Weinstein and C. Zhu, Integration of twisted Dirac brackets. Duke Math. J. 123 (2004), 549-607.

[11] H. Bursztyn and O. Radko, Gauge equivalence of Dirac structures and symplectic groupoids, Ann. Inst. Fourier (Grenoble) 53 (2003), 309-337.

[12] A. Cattaneo and P. Xu, Integration of twisted Poisson structures, J. Geom. Phys. 49 (2004), $187-196$.

[13] T. Courant, Dirac manifolds, Trans. A.M.S. 319 (1990) 631-661.

[14] D. Iglesias Ponte, C. Laurent-Gengoux and P. Xu, Universal lifting theorem and quasi-Poisson groupoids, preprint (2005), math.DG/0507396.

[15] D. Iglesias Ponte and P. Xu, Hamiltonian spaces for Manin pairs over manifolds, Arxiv:0809.4070 [math.DG].

[16] Z.-J. Liu, A. Weinstein and P. Xu, Manin triples for Lie algebroids. J. Differential Geom. 45, (1997), 547-574.

[17] J.-H. Lu, Momentum maps and reduction of Poisson actions, Symplectic geometry, groupoids and integrable systems (Berkeley, CA, 1989), 291-311. Springer, New York, 1991.

[18] K. Mackenzie, General theory of Lie groupoids and Lie algebroids, London Math. Soc. Lecture Notes Series 213. Cambridge University Press, Cambridge, 2005.

[19] K. Mackenzie and P. Xu, Lie bialgebroids and Poisson groupoids, Duke Math. J. 73 (1994) 415-452.

[20] D. Roytenberg, Courant algebroids, derived brackets and even symplectic manifolds, U. C. Berkeley thesis, 1999. Arxiv:math.DG/9910078.

[21] — Quasi-Lie bialgebroids and twisted Poisson manifolds, Lett. Math. Phys. 61 (2002) 123-137.

[22] __ On the structure of graded symplectic supermanifolds and Courant algebroids, In: Quantization, Poisson Brackets and Beyond, Theodore Voronov (ed.), Contemp. Math. 315, Amer. Math. Soc., Providence, RI, 2002.

[23] P. Ševera, Letters to A. Weinstein, avail. at http://sophia.dtp.fmph.uniba.sk/ severa/letters/.

$[24]$, Noncommutative differential forms and quantization of the odd symplectic category, Lett. Math. Phys. 68 (2004), no. 1, pp. 31-39, 2004.

[25] _ Some title containing the words "homotopy" and "symplectic", e.g. this one, Travaux Mathématiques XVI (2005), arXiv:math/0105080.

[26] P. Ševera and A. Weinstein, Poisson geometry with a 3-form background, Prog. Theo. Phys. Suppl. 144 (2001), 145-154.

[27] P. Xu, Morita equivalence and momentum maps, J. Differential Geom. 67 (2004) 289-333. 
Instituto Nacional de Matemática Pura e Aplicada (IMPa), Estrada Dona Castorina 110, Rio de Janeiro, 22460-320, Brazil

E-mail address: henrique@impa.br

Instituto de Ciencias Matemáticas (CSIC), C/ Serrano 113bis, Madrid, 28006, Spain

E-mail address: iglesias@imaff.cfmac.csic.es

Section de Mathématiques, Université de Genève, 2-4 Rue du Lièvre, Case postale 64, CH-1211 Genève 4, Switzerland, on leave from FMFI UK, Bratislava, Slovakia

E-mail address: pavol.severa@gmail.com 